\title{
SKIN MANIFESTATIONS OF GASTROINTESTINAL DISEASES: A REVIEW
}

Manisha Nijhawan ${ }^{1}$, Puneet Agarwal2 ${ }^{2}$, Sandeep Nijhawan ${ }^{3}$, Prashant ${ }^{4}$, Abhishek Saini ${ }^{5}$

\section{HOW TO CITE THIS ARTICLE:}

Manisha Nijhawan, Puneet Agarwal, Sandeep Nijhawan, Prashant, Abhishek Saini. "Skin Manifestations of Gastrointestinal Diseases: A Review". Journal of Evolution of Medical and Dental Sciences 2014; Vol. 3, Issue 09, March 3; Page: 2357-2372, DOI: 10.14260/jemds/2014/2146

ABSTRACT: Skin is the largest organ of human body and stands as a guard for our internal organs. It can be regarded as a mirror giving a reflection of metabolic, biochemical and functional status of our internal organs. Dermatologists/Gastroenterologist should be aware of the dermatological manifestations as these change may be the first clue that a patient has underlying gastrointestinal (GI) or liver disease. Recognizing these signs is important in early and appropriate diagnosis. This article reviews the important dermatological manifestation of various GI and liver diseases.

KEYWORDS: Skin and GI.

Different dermatological manifestation in gastrointestinal diseases can be classified as:-

1) Specific skin manifestations

2) Reactive skin manifestations

3) Skin manifestations secondary to the deficiency of nutrients due to GI disease

4) Skin manifestations secondary to the treatment

For clinician skin manifestation can be simply classified as-

1. Dermatological manifestations in benign GI diseases

2. Dermatological manifestations in malignant GI disease

\section{Dermatological manifestation of gastrointestinal diseases}

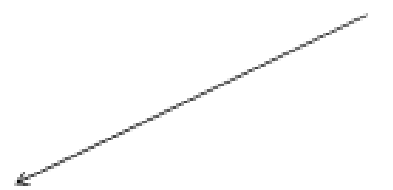

Cutaneous manifestation in nonmalignat gastrointestinal diseases

1. Cutaneous manifestation in esoophageal diseases

2. Cutaneous manifestation in gastric diseases

3. Cutaneous manifestation in small intestinal diseases

4. Cutaneous manifestation in large intestinal disease

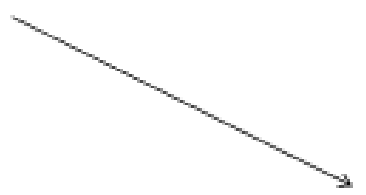

Cutaneous manifestation in malignant gastrointest inal disease

1. Cutaneous metastatic disease

2. Paraneoplastic dermal manifestation

3. Cutaneous manifestation due vasoactive substance producing tumor

4. Cutaneous manifestation in GI polyposis 


\section{A: Skin manifestations in esophageal diseases: \\ Dysphagia:}

Esophageal webs

This is a developmental abnormality with one or more horizontal membrane in upper esophagus. These patients may have associated koilonychia, iron deficiency anemia, angular stomatitis and dysphagia which are described as Plummer Vinson syndrome. ${ }^{1}$

Collagen vascular diseases

Dysphagia can be the only presentation in systemic sclerosis and dermatomyositis. ${ }^{2,3}$

Bullous disorders

Bullous disorders like pemphigus, Ciccatrical pemphigoid, Bechet's disease and epidermolysis bullosa can present as dysphagia. ${ }^{4}$

Infections

Infections like candidiasis and herpes can involve both esophagus and skin.

Miscellaneous

Lichen planus

\section{B: Skin manifestations in gastric disease:}

H.pyloric gastritis: Cutaneous manifestations associated with $\mathrm{H}$. pylori are scleroderma, acne rosacea, idiopathic urticaria, which occurs due to immune mediated reaction as a result of cross reactivity between bacteria and host. ${ }^{5}$ Sjogren's syndrome, Lichen planus, Bechet's disease and Henoch schonlein purpura can be seen in some of the patients. ${ }^{6}$

Sweet syndrome is also associated which is characterized by fever, peripheral neutrophilic leukocytosis, painful erythematous juicy plaques and nodules over extremities, head and neck. On histopathology dense neutrophilic infiltrate in the superficial dermis and edema of papillary dermis is seen. ${ }^{7}$

H. Pylori infection is also associated with Atopic dermatitis and Prurigo nodularis. 8

\section{C: Skin manifestations in small intestine diseases:}

1. Celiac disease: $C D$ is characterized by intestinal malabsorption with total or subtotal villous atrophy which improve with gluten free diet. ${ }^{9} \mathrm{CD}$ is associated with various skin diseases. Several hypotheses have been proposed regarding cutaneous manifestations. An abnormal small intestinal permeability appears to be implicated, which may allow the crossing of endogenous or exogenous antigens and may provoke the immunological response. ${ }^{10}$ 


\begin{tabular}{|l|}
\hline Skin disorders in celiac disease \\
\hline Dermatitis herpetiformis \\
Linear IgA bullous dermatosis \\
Urticaria \\
Hereditary angioneurotic edema \\
Cutaneous vasculitis \\
Erythema nodosum \\
Erythema elevatum diutinum \\
Necrolytic migratory erythema \\
Psoriasis \\
Vitiligo disease \\
Behçet's disease \\
Oral lichen planus \\
Dermatomyositis \\
Porphyria \\
Alopecia areata \\
Acquired \\
lanuginosa \\
Ichthyosiform dermatoses \\
Pellagra \\
Generalized acquired cutis laxa \\
Atypical mole syndrome and \\
congenital \\
giant nevus \\
Pyoderma gangrenosum \\
\hline
\end{tabular}

Dermatitis herpetiformis: Dermatitis herpetiformis (DH) presents as an itchy, chronic, papulovesicular eruption which may leave pigmentation and scarring. ${ }^{11}$ skin lesions are characterized by a symmetrical eruption on the extensor surfaces of the body. DH tends to occur more in the adult male (M/F ratio 2:1). Histology shows micro abscesses consisting of neutrophils and eosinophils within the dermal papillae. Direct immunofluorescence of the normal skin shows the pathognomonic granular IgA deposits in the papillary dermis. ${ }^{12}$ Antigluten, antiendomysium, antigliadin and tissue transglutaminase antibodies have been detected in patients with DH. The DH rash is often treated with dapsone in conjunction with the gluten withdrawal. ${ }^{13}$

Urticaria: Urticaria is a transient erythematous or edematous swelling of the dermis or subcutaneous tissue and is due to a local increase of permeability of capillaries and small venules. Hautekeete et al first described the association between CD and chronic urticaria, although this association is still debated.14 
Hereditary angioneurotic edema: It is characterized by recurrent edematous attacks in the subcutis and sub-mucous due to C1-esterase inhibitor (C1-INH) deficiency]. In 2002, Farkas et al first described the simultaneous occurrence of HANE and CD. ${ }^{15}$

Cutaneous vasculitis: The coexistence of cutaneous vasculitis and CD might be related to increased intestinal permeability ${ }^{16}$. Meyers et al described a case of cutaneous vasculitis complicating CD and the remission of skin lesions after the treatment with a strict GFD. ${ }^{17}$

Erythema nodosum: Erythema nodosum (EN) is a chronic panniculitis characterized by inflammation of the fat septa. In 1991, Durand et al first observed a causal linkage between CD and EN. ${ }^{18}$

Psoriasis: Psoriasis is a chronic, relapsing dermatosis characterized by scaling and erythema. Recent studies have showed an association between $\mathrm{CD}$ and psoriasis and an improvement of skin lesions after 3-6 mo of GFD. In a screening study conducted by Michaelsson et al, 16\% of 302 subjects with psoriasis had IgA and/or IgG AGA. ${ }^{19}$

2. Gastrointestinal hemorrhage: Patient presenting with gastrointestinal bleed, skin examination can help to rule out important clinical conditions and deciding the management. Some of the dermatological disorders associated with these conditions are described below

Hereditary hemorrhagic telangiectasia (Osler-Weber-Rendu disease): Recurrent painless gastrointestinal bleeding occurs from vascular malformations throughout gastrointestinal tract. Bleeding is chronic, periodic, progressive and massive. Lesions usually appear in first few years of life and recurrent epistaxis is characteristic but, hematemesis is less common. Visible cutaneous and mucosal telangiectasia on lips, nasal mucosa, tongue, palate, palms and soles, finger tips are seen. Site of bleeding may be visualized on endoscopy. Angiography may identify large arterio-venous malformation. ${ }^{20}$

Skin lesions consist of small macular telangiectasia which often increases in number and size with age. ${ }^{21}$ Telangiectasia usually appear over lips, tongue palate face ears conjunctive chest hand and feet. Estrogen and aminocaproic acid can be used for treatment and in some cases endoscopic treatment can be tried. ${ }^{20}$

Blue rubber bled nevus syndrome: Rare disorder characterized by large cutaneous hemangiomas and gastrointestinal bleeding from vascular malformation. It is usually identified at birth or early childhood.22,23 Four types of skin lesions have been described which in contrast to classical hemangiomas do not regress spontaneously. ${ }^{24}$

1. Blue rubber nipple $\rightarrow$ most characteristic cutaneous lesions described as soft, rubbery, compressible nodule which after being compressed leaves an empty bluish sac that swiftly refills with blood.

2. Tender, irregular, blue-black, punctuate macules distributed on the extremities and trunk.

3. Large disfiguring hemangiomas that may interfere with vital limb or organ function.

4. Segmental hemangiomas have also been found to be associated with visceral haemorrhage. 
Kaposi sarcoma: Neoplasm of vascular endothelium and pericapillary cells that often presents on the skin and may involve other visceral organs including gastrointestinal tract. Gastrointestinal tract is involved in $50 \%$ to $80 \%$ of those with the cutaneous lesions and in nearly $100 \%$ of those with oral lesions. ${ }^{25}$ Herpes virus type 8 is involved in pathogenesis of Kaposi's sarcoma. ${ }^{26}$ Cutaneous lesions are characterized by multiple red purple macules which change into deeper violet papule or plaque. Lesions can appear anywhere on skin with a propensity for legs and feet in the classic non-HIV form. Disseminated cutaneous lesions are found in acquired immunodeficiency syndrome (AIDS) related Kaposi's sarcoma.

Pseudoxanthoma Elasticum: It is a genetic disorder of elastic fibres that become calcified and weakened. It is characterized by specific skin lesions, angioid streaks and other degenerative changes of the retina and widespread vascular calcification leading to gastrointestinal bleeding, premature myocardial infarction, cerebrovascular disease, peripheral vascular disease and hypertension. ${ }^{27}$

The skin findings includes small, yellow, papules coalescing to form plaques with a plucked chicken or cobble stone appearance. These are mainly distributed on flexural areas including neck and axilla. In addition, generalized laxity of face, neck and trunk may be found. Endoscopy may reveal the characteristic yellow cobblestone like lesions similar to those occurring in the mouth and skin. ${ }^{28}$

Ehlers-Danlos syndrome: This is a hereditary collagen disease characterized by fragility of the skin and blood vessels, hyperextensibility of joints and hyperelasticity of the skin. Type IV Elhers danlos syndrome is the ecchymotic type and is most closely associated with gastrointestinal hemorrhage. ${ }^{29}$

Henoch Schonlein purpura: Gastro intestinal bleeding can be major feature. Patients have palpable purpura on skin, leukocytoclastic vasculitis, edema and hemorrhage. ${ }^{30}$

Malignant atrophic Papulosis (Degos' disease): It is a rare disorder of the young adult affecting the skin, gastrointestinal tract and central nervous system. The characteristic skin lesions are erythematous multiple papules undergo umblication leading to atrophic white scar like centers surrounded by an erythematous border. The skin lesions often precede the development of infracts involving the gastrointestinal and central nervous systems. Gastrointestinal infarctions may cause gastrointestinal bleeding or perforation and fatal peritonitis in $50 \%$ cases. $^{31}$

\section{Skin in non-malignant large intestinal disorders:}

Inflammatory bowel disease: Ulcerative colitis and Crohn's disease: Fissures and fistulas are the most frequent cutaneous manifestations occur mainly in crohn's disease. These appear mainly on perianal region, but can occur on stomas or the abdominal wall. Perianal fissures are usually multiple and may lead to ulcer or abscesses. Crohn's disease may also affect the oral mucosa as represented by a cobblestone pattern, ulceration and nodules. ${ }^{32}$

Metastatic Crohn's disease present as nodules, plaques or ulcerated lesions on skin that is histopathologically identical to crohn's disease. They may be located on any area of skin and are often correlated with disease activity. Sarcoidal type granulomatous histology can be seen in the skin or mucosa and correlates to disease activity. ${ }^{33}$ 
Erythema Nodosum presents as tender red nodules on the anterior aspect of the leg. It may occur at any point in the course of inflammatory bowel disease or may be associated with flare of enteric disease. ${ }^{34}$

Oral Apthous Ulcers are found in association with inflammatory bowel disease and reflects disease activity but, these lesions are nonspecific. ${ }^{35}$

Pyoderma Gangrenosum usually begins as a hemorrhagic pustule or papule after minimal trauma and rapidly evolves into a purulent, painful, necrotic ulcer with dusky undermined borders. Lesions are sterile and heal with scarring. Fifty percent of the patients with Pyoderma gangrenosum have ulcerative colitis. The eruption typically occurs on the trunk. Histological findings may vary, demonstrates mild to moderate perivascular lymphocytic infiltrate and endothelial swelling and dense neutrophilic infiltrate in the dermis. Pyoderma vegetans can also complicate the disease. ${ }^{36}$

Sweet syndrome is characterized by acute febrile neutrophilic dermatosis. Patient develops erythematous, tender papules, plaques and nodules. ${ }^{37}$

Ten percent patients of inflammatory bowel disease develop thrombophlebitis as it is associated with hypercoagulable state. Cutaneous gangrene can also develop. ${ }^{38}$

Some patients may develop granulomatous chelitis.

\section{Cutaneous manifestation in inflammatory bowel disease}

1. Fissures and fistulas are the most frequent in Crohn's disease

2. Metastatic Crohn's disease

3. Erythema Nodosum

4. Oral Apthous Ulcers

5. Pyoderma Gangrenosum

6. Sweet syndrome

7. Thrombophelibitis

8. Granulomatous chelitis

\section{Skin manifestation in hepatic disorders:}

Chronic liver disease: There are various skin manifestations in patients of chronic liver disease.

1. Icterus - Yellowish discoloration of skin and sclera and visible when serum bilirubin level exceeds $2.5-3 \mathrm{mg} / \mathrm{dl}$

2. Hyperpigmentation

3. Spider nevus

4. Palmer erythema

5. Pruritus (associated with cholestasis)

6. Sparse axillary and pubic hairs

7. Gynecomastia

8. Clubbing

9. Terry's nail- whitening of nail plate except lunula

10. Muehreke's nail

11. Splinter hemorrhage

12. Bluish lunula in Wilson's disease 
Hepatitis A Virus: Jaundice rarely cryoglobulinemia and vasculitis. ${ }^{39}$

Hepatitis B Virus: Cutaneous manifestation can be present in upto $50 \%$ patients. Utricaria can be seen in 5-7 \%. Skin rashes in chronic HBV are more likely to have palpable purpura, which histologically is a neutrophilic necrotizing vasculitis involving small vessels. ${ }^{40}$ An association between HBV and polyarteritis nodosa has been well established. ${ }^{41}$ Palpable purpura, non-blanching purple nodules usually first appear on the lower limbs or dependent areas and spread centrally. Another form of immune complex disease is cryoglobulinemia. The presence of cryoglobulins in patients with persistent HBV infection has been estimated as high as 15\%. The cryoprecipitate is primarily of the mixed form. These symptoms are frequently associated with Raynaud's phenomenon and may be exacerbated by cold. ${ }^{42}$

Papular acrodermatitis of childhood (PAC) is a cutaneous disease reported to be caused by chronic HBV, but this is unsubstantiated. This disease has also been called 'Gianotti-Crosti Syndrome. 'It is most prevalent in the Mediterranean region. Histology reveals a superficial perivascular lymphophagocytic infiltrate with mild endothelial swelling. However, there are no clear histological features of vasculitis and no conclusive evidence of HBsAg deposition in the skin. Clinically, this disorder is characterized by nonpruritic erythematous, $2-3 \mathrm{~mm}$ popular eruptions in children, primarily of the face and extremities; lymphadenopathy (inguinal and axillary); and anicteric acute hepatitis found in association with HBsAg. Usually, the children are ages 2-6 years, rarely older than age 10 years. Anti-HBs is not detected during the dermatitis phase, but peaks 6-12 months later; hence, HBsAg has been postulated to play a role in recovery. ${ }^{43}$ Frequency of cutaneous manifestation in a prospective study by Cacoub et al in France was upto 17\%.

\section{Cutaneous manifestation hepatitis B}

1. Papules within 24 hours in acute hepatitis B

2. Popular acrodermatitis

3. Neutrophilic necrotizing vasculitis

Hepatitis C Virus: Chronic hepatitis C infection is associated with many extra hepatic manifestations in joints, muscles, neural and gastrointestinal tissues, and skin. Dermatologic manifestations of hepatitis $\mathrm{C}$ virus (HCV) are classified according to diseases with proven or suspected etiology or causation.

Primary causation results from direct infection of HCV in the skin, lymphocytes, dendritic antigen-presenting cells, and blood vessels. An example of this type of disorder is the recent finding of epidermal cells with HCV-RNA particles.

Secondary causation occurs when HCV infection manifests in the skin due to epiphenomena resulting from the disruption of immune responses. Leukocytoclastic vasculitis due to cryoglobulinemia is a good example of a specific skin manifestation resulting from the production of immunoglobulins, with rheumatoid characteristics causing an immune complex-mediated vasculitis. HCV has been associated with cryoglobulinemia, primarily of the mixed (type II or III) forms. ${ }^{44} \mathrm{HCV}$ RNA can be found by polymerase chain reaction in the serum in upto $85 \%$ of patients with mixed cryoglobulinemia and in $90 \%$ of their cryoprecipitates. Some authors suggest that HCV may be the 
leading cause of essential mixed cryoglobulinemia and infection with HCV should be evaluated in all patients presenting with this syndrome. Polyarteritis nodosa is also noted in patients with HCV, although not frequently. 45

Tertiary causation of dermatologic manifestations results when the disruption of another organ infected or affected by HCV causes skin manifestations that are nonspecific and typical of skin responses to that organ; these responses result from a wide range of causes, including flushing and other findings of thyroid hormone release in early HCV-linked autoimmune thyroiditis. Chronic active hepatitis leading to fibrotic liver disease in chronic hepatitis $\mathrm{C}$ infection can also cause cutaneous vascular changes, such as spider nevus and palmar erythema. Arteriovenous hemangioma, a benign acquired cutaneous vascular lesion, has also been reported to be associated with chronic liver disease, including chronic active hepatitis associated with HCV infection.

Another category of dermatologic manifestations in HCV infections in a causative schema includes those diseases in which an association has been identified, but the details of causation have not yet been clarified. Porphyria cutanea tarda (PCT) is a good example of this type of HCV-related disease in which causation is unexplained but undeniable. In patients with PCT, $70 \%$ are HCV positive. Lichen planus is also associated with HCV.46, 47 Necrolytic acral erythema is almost exclusively seen in HCV infection. Red finger syndrome is characterized by telangiectasia of finger and toe is also rarely seen.48

Neoplastic dermatologic manifestations are another category of extra hepatic findings.

Dermatologic manifestations are associated with treatments of HCV infection, especially interferon. The most striking of these changes is cutaneous necrosis at the site of interferon injection.

The last category is suspected associations of the disorder in a causative schema. HCV genomic analysis by means of arduous gene sequencing of many viruses has led to the division of HCV into genotypes based on homology. Arabic numerals denote the genotype, and a letter denotes the subtype for lesser homology within each genotype. ${ }^{49}$

\section{Cutaneous manifestation hepatitis C}

1. Direct infection of HCV in the skin

2. Leukocytoclastic vasculitis due to immune response

3. Disruption of another organ infected or affected by HCV causes skin manifestations

4. Dermatologic manifestations are associated with treatments of HCV infection

Primary biliary cirrhosis (PBC): Xerosis and melanosis is also seen in upto half of patients. Xanthoma and xanthelasma can be seen in one third of patients. ${ }^{50}$ Lichen planus has been associated with a number of liver diseases, including primary biliary cirrhosis. CREST syndrome associated with PBC is known as Reynold's syndrome. 51

Alcoholic liver disease: Cutaneous lesions often accompany alcoholic liver disease and have been found in up to $43 \%$ of people with chronic alcoholism. ${ }^{52}$ The combined prevalence of palmar erythema, spider angiomas, and Dupuytren's contracture in alcoholic cirrhosis is found to be $72 \%$. Paper-money skin and Dupuytren's contracture are more distinct lesions for alcoholic cirrhosis. ${ }^{53}$ Recognizing these skin changes contribute to the diagnosis and staging of liver cirrhosis. ${ }^{54}$ 
Cholestatic liver disease: In cholestatic liver disease pruritus occur due to retention of bile acids which act as hepatotoxins, although a direct correlation between blood or tissue level of bile acids and pruritis has not been made. Shiny nails can be seen due to intense pruritis. Long term cholestasis can lead to formation of skin xanthomas. 55,56

Chronic liver disease: Pigmentary changes which includes yellowish discoloration of the skin and mucous membrane due to accumulation of bilirubin and its metabolite. The skin color may vary from light yellow to dark green depending on the bilirubin level and patient's own skin color.

The cutaneous vasculature is also frequently affected in liver disease. The most recognized vascular lesions are spider angiomas. These lesions most often occur on the face, upper trunk and distal upper extremities. They are formed by a central vessel surrounded by branching small vessels. Compression of the central punctum results in blanching of the entire lesion. The spider angiomas can be treated with fine cautery or pulse dye laser. Palmer erythema may present clinically as increased mottling of the hands or discrete erythematous patches on the thenar or hypothenar eminences. Both of these lesions have been associated with faulty metabolism of estrogen by a diseased liver.

Hemochromatosis: Hemochromatosis or "bronze diabetes" is a devastating multisystem disease. It is among the most common genetic disorders of metabolism, and results in deposition of iron in tissues and organs throughout the body, including the liver, usually in patients ages 30 to 40 .

Surplus iron stores can result in widespread bronze discoloration of skin exposed to the sun. Hemochromatosis also results in loss of body hair, ichthyosis and koilonychia. ${ }^{57}$

Wilson's disease: Most prominent cutaneous abnormality associated with this condition is azure lunulae, (bluish discoloration of the white half-moon) at the proximal portion of the nail bed. The discoloration is limited to the lunula, differentiating the nails of hepatolenticular degeneration from diseases in which the entire nail bed turns blue. These conditions include Argyria, pueudomonal infections and in patients on antimalarial. In some patients adverse reaction due to pencillamine which is used in treatment of Wilson's disease can lead to rashes, pemphigus and elastosis perforans serpiginosa. 58

Skin manifestation in pancreatitic diseases: Acute pancreatitis can be associated with Turner's sign (periumbilical ecchymosis) and Cullen's sign (ecchymosis of flank) which are both caused by extravasation of hemorrhagic peritoneal fluid into the skin. Pancreatic panniculitis is a rare lobular panniculitis (inflammation of the subcutaneous fat lobules with fat necrosis and calcification) associated with pancreatitis or pancreatic carcinoma. Lesions consist of tender, erythematous subcutaneous nodules occurring on distal extremities, often periarticular. This type of lobular panniculitis has characteristic histologic features of basophilic degeneration of lipocytes leading to formation of ghost cells, calcification and saponification of the dermal collagen and necrosis. ${ }^{59}$

Skin manifestation of gastrointestinal malignancy: Various gastrointestinal malignancies may be associated with various cutaneous manifestations which may be presenting sign of these diseases. They are usually seen in advance stage of disease. In some cases the skin can be directly infiltrated by 
cancer cells that represent metastatic spread from a GI malignancy (e.g. a Sister Mary Joseph nodule). In other cases, the skin lesions are related to the underlying presence of malignancy, but they do not contain malignant cells and are referred to as paraneoplastic dermatological syndromes

Cutaneous Metastatic Disease: Cutaneous metastatic disease from gastrointestinal tract neoplasms is most often found on abdominal skin and scalp. Metastatic cancer to the skin is characterized by one or more firm, indurated and often ulcerated nodules or plaques. These nodules may be dermal or subcutaneous. Sister Mary Joseph's nodules describes firm and indurated nodules over umbilicus, is a sign of metastatic disease. Adenocarcinoma of gastrointestinal tract most commonly stomach is the common primary site. ${ }^{60}$

Paraneoplastic dermatological syndromes: Acanthosis Nigricans is classic example of paraneoplastic syndrome. The disease initiates with symmetric skin hyperpigmentation in the axillary and inguinal folds, submammary region, around the mammilla, umbilical, and ano-genital regions. The neck region is frequently affected in childhood. Similar pigmented papillomatous lesions can be observed on the mucous membranes of the oral cavity, nasal, and laryngeal mucosa, and vulva. Malignancy-associated acanthosis nigricans is frequently seen with GI cancers. 61,62

Leser-Trélat or Leser-Trélat syndrome (LTS) is an eruptive appearance of, or at least a sudden increase in, the number or size of multiple seborrheic keratoses in association with an internal malignancy. The majority of patients with LTS have adenocarcinomas, most commonly of the stomach, colon or rectum and less frequently carcinomas of esophagus, duodenum or hepatocellular carcinoma. ${ }^{63}$

Tylosis Palmaris et Plantaris (Howel Evans syndrome) describes a familial association of punctuate keratosis of the palms and soles with esophageal carcinoma. ${ }^{64}$

Tripe palms is a paraneoplastic phenomenon characterized by velvety and cobbled surface of palm and seems to be associated with gastric neoplasm. 65

Dermatomyositis is characterized by violaccous color of eyelid (heliotrope), keratotic papules over knuckes (Gottron's papule), erythema over knee/elow (Gottron's sign). About 1/4th of the patients are associated with internal malignancy mainly gastric and colorectal cancer. ${ }^{66}$

Cutaneous manifestation by tumors producing vasoactive substance: Carcinoid syndrome is caused by tumors producing vasoactive substances including serotonin, kallikrein and histamine. The clinical manifestations include paroxysms of flushing, wheezing, diarrhea, abdominal pain and often hypotension. Symptomatic carcinoid syndrome usually indicates metastasis to liver. Diagnosis is made by detecting 5-hydroxyindoleacetic acid in urine. ${ }^{67}$

Glucogonoma a tumor of the pancreatic alpha cells is associated with necrolytic migratory erythema called glucogonoma syndrome. Necrolytic migratory erythema is a distinctive eruption characterized by recurrent cycles of erytheme followed by blisters, erosions, crusting and scaling on the lower abdomen, groin and buttocks. Other cutaneous manifestations of glucagonoma syndrome include angular cheilitis and painful glossitis. 68 


\section{REVIEW ARTICLE}

\section{Dermatological manifestation associated with gastrointestinal polyposis:}

Gardner's syndrome: Gardner's syndrome refers to the clinical triad of adenomatous polyps of the gastrointestinal tract with a high risk of malignant transformation, epidermal cysts and dermoid tumors. 69

The skin and bone lesions occur before the intestinal polyps and may predate the polyps by as many as 10 years. Epidermal cysts are multiple, appear before puberty and are located predominantly on extremities, face and scalp. Desmoid tumors occur in young patients with female predominance, are locally aggressive and do not metastasize but can lead to death. Gardner's syndrome also has an association with fibromas, lipomas, dental abnormalities, pigmented ocular fundal lesions, brain tumors and endocrine tumors. ${ }^{70}$

Peutz-Jeghers syndrome: Peutz-Jeghers syndrome (PJS) is an autosomal dominant disorder. The syndrome is caused by mutations in STK11/LKB1.Cutaneous lesions consist of 1-5 mm diameter hyperpigmented macules, irregularly distributed over the buccal mucosa, gums, hard palate and lips, mainly on the lower lip. Lentigines usually appear during early childhood, and have a tendency to increase in size. Larger maculae (melanosis) are rarely seen over the back of the hands, the tips of the fingers and toes, and over the palms and soles. ${ }^{71}$

GI tract manifestations include numerous intestinal polyps in the jejunum, ileum and less frequently in the colon, rectum, stomach and duodenum that are typical hamartomas ${ }^{72}$

Canada-Cronkhite syndrome: Cutaneous lesions in patients with Cronkhite-Canada syndrome (CCS) include hyperpigmented macules ranging from a few millimeters to $10 \mathrm{~cm}$ in diameter, localized on the dorsal surface of the hands, palms, arms, neck, face and scalp. Fingernails and toenails have discoloration, atrophy, nail plate separation and shedding. Alopecia occurs rapidly and in some cases leads to total hair loss. Patients present with abdominal cramps, diarrhea and weight $\operatorname{loss}^{73}$

GI lesions in CCS are hamartomatous polyps histologically revealing pseudopolypoidinflammatory changes. Although considered a benign condition, in 1967, Gomes da Cruz reported an association of this syndrome with a cancer of the cecum and descending colon ${ }^{74}$

Cowden's disease (mulptiple hamartomas syndrome): It is an autosomal dominant disorder with variable expression. It is characterized by mucocutaneous lesions, grastrointestinal tract polyps. The mucocutaneous lesions include facial trichilemmomas (flesh coloured, flat topped or elongated verrucous papules in a periorificial and centrofascial distribution), oral papillomatosis (small, smooth, flesh colored papules on labial, gingival or palatal surfaces resulting in cobblestone appearance), Cowden's fibroma(firm, white papules or nodules involving the face, scalp or hands), acral keratosis(flesh colored, flat topped, smooth or rough papules on dorsum of hand and feet that may resemble common warts) and palmoplantar keratosis(firm hyperkeratotic papules). Less common skin findings include lipomas, hemangiomas, scrotal tongue and neuromas ${ }^{75,76}$

Muir-Torre Syndrome: Muir-Torre syndrome (MTS) is defined by the development of internal malignancy, most commonly colon cancer, in association with sebaceous adenomas and epitheliomas, 
sebaceous carcinomas and multiple or early-onset keratoacanthomas. Sebaceous adenoma is believed to be the most specific lesion of Muir-Torre syndrome.77, 78

Cancers of the GI tract comprise more than $60 \%$ of the visceral malignancies in MTS, and colorectal cancer is the predominant neoplasm. Tumors are located predominantly in the proximal colon, in contrast to the general populace, whose colorectal tumors are usually distal to the splenic flexure.

\section{Miscellaneous:}

Acrodermatitis enteropathica: This is an autosomal recessive disease with defect in zinc absorption. It is characterized by diarrhea with periorificial and acral erythematous vesiculopustular eruptions ${ }^{79,80}$.

\section{Infectious:}

1. Cutaneous amoebiasis

2. Ground itch due to hook worm

3. Larva currens due to strongyloides

4. Pruritus ani

\section{Congenital diseases:}

1. Hereditary hemorrhagic telengietasia

2. Porphyria

3. Tuberous sclerosis

\section{Acquired diseases:}

1. Acute graft versus host disease

2. Langerhans cell histocytosis

3. Systemic mastocytosis

4. Systemis amyloidosis

5. Vinyl chloride disease

\section{REFERENCES:}

1. Novacek G. syndrome". Orphanet J 2006; Rare Dis 1: 36. doi:10.1186/1750-1172-1-36.

2. C N Rajapakse, J Bancewicz, C J Jones et al. Pharyngo-oesophageal dysphagia in systemic sclerosis. Ann Rheum Dis 1981 40: 612-614.

3. Ertekin C, Seçil Y, Yüceyar N, Aydoğdu I. Oropharyngeal dysphagia in polymyositis/dermatomyositis. Clin Neurol Neurosurg. 2004 Dec;107(1):32-7.

4. Bübl R, Schön B. Dysphagia in dermatologic disease. Dysphagia. 1993;8(2):85-90.

5. Hernando-Harder AC, Booken N, Goerdt S et al. Helicobacter pylori infection and dermatologic diseases. Eur J Dermatol 2009.

6. Wedi B, Kapp A. Helicobacter pylori infection in skin diseases: a critical appraisal. Am J Clin Dermatol 2002; 3: 273-82.

7. Di Campli C, Gasbarrini A, Nucera E, et al. Beneficial effects of Helicobacter pylori eradication on idiopathic chronic urticaria. Dig Dis Sci 1998; 43:1226-1229. 
8. Tebbe B, Geilen CC, Schulzke JD, et al. Helicobacter pylori infection and chronic urticaria. J Am Acad Dermatol 1996;34:685-686.

9. Corazza GR, Gasbarrinin G. Coeliac disease in adults, Baillieres Clin Gastroenterol 1995; 9: 329350.

10. Heyman M, Abed J, Lebreton C, Cerf- Bensussan N. Intestinal permeability in celiac disease: insight into mechanisms and relevance to pathogenesis. Gut.2012 Sep;61(9): 1355-64.

11. Farrell RJ, Kelly CP. Celiac spru. N Engl J Med 2002; 346:180-188.

12. Seah PP, Fry L, Holborow EJ, Rossiter MA, Doe WF, Magalhares AF, Hoffbrand AV. Antireticulin antibody: incidence and diagnostic significance Gut 1973; 14:311-315.

13. Seah PP, Fry L, Hoffbrand AV, Holborow EJ. Tissue antibodies in dermatitis herpetiformis and adult celiac disease. Lancet 1971; 1:834-846.

14. Gabrielli M, Candelli M, Cremonini F, Ojetti V, Santarelli L, Nista EC et al. Idiopathic chronic urticaria and celiac disease. Dig Dis Sci 2005; 50:1702-1704.

15. Menzies IS, Laker MF, Pounder R, Bull J, Heyer S, Wheeler PC, Creamer B. Abnormal intestinal permeability to sugars in villous atrophy. Lancet 1979; 2:1107-1109.

16. Meyers S, Dikman S, Spiera H, Schultz N, Janowitz HD. Cutanuous vasculitis complicating celiac disease. Gut 1981; 22:61-64.

17. Woo WK, McMillan SA, Watson RG, McCluggage WG, Sloan M, McMillan JC. Coeliac diseaseassociated antibodies correlate with psoriasis activity. Br J Dermatol 2004; 151:891-894.

18. Reilly PJ, NOstrant TT. Clinical manifestations of hereditary hemorrhagic telangiectasia. Am J Gastroenterol 1984;79:363-367.

19. Perry WH. Clinical spectrum of hereditary hemorrhagic telangiectasia (Osler - Weber-Rendu Disease). Am J Med 1987; 82:989-997.

20. Gregory B, Ho VC. Cutaneous manifestations of gastrointestinal disorders: Part II. J Am Acad Dermatol 1992; 26:371-383.

21. Oranje AP. Blue rubber bleb nevus syndrome. Pediatr Dermatol 1986; 3:304-310.

22. Lamberts RJ. Gastrointestinal hemorrhage and the skin. Dermatol Clin 1989; 7:403-415.

23. Guarda LA, Luna MA, Smith JL et al. Acquired immunodeficiency syndrome: Post-mortem findings Am J Clin Pathol 1986; 81:549-557.

24. Moore PS, Chang Y. Detection of herpes virus-like DNA sequences in Kaposi's sarcoma in patients with and without HIV infection. N Engl J Med 1985; 332:1181-1185.

25. Callen JP. Skin manifestations of digestive disease. In Berk EJ (ed): Gastroenterology. Philadelphia, WB Saunders, 1995, pp 3324-3344 9-3

26. Neldner KH. Pseudoxanthoma elasticum. Int J Dermatol. Mar 1988;27(2):98-100

27. Byers PH, Murray ML. Heritable collagen disorders: the paradigm of Ehlers-Danlos syndrome. Journal of Investigative Dermatology 2012; 15 (132): E6-11.

28. Kraft DM, Mckee D, Scott C. Henoch-Schönlein purpura: a review". American Family Physician 1998; 58 (2): 405-8, 411.

29. High WA, Aranda J, Patel SB, Cockerell CJ, Costner MI. Is Degos' disease a clinical and histological end point rather than a specific disease? J Am Acad Dermatol Jun 2004; 50 (6): 8959.

30. Gagoh OK, Qureshi RM, Hendrickse MT. Recurrent buccal space abscesses: a complication of Crohn's disease. Oral Surg Oral Med Oral Pathol Oral Radiol Endod 1999; 88:33-36. 
31. Parks AG, Morson BC, Pegum JS. Crohn's disease with cutaneous involvement. Proc R Soc Med. $1965 ; 58: 241-242$.

32. Su CG, Judge TA, Lichtenstein GR. Extraintestinal manifestation of inflammatory bowel disease. Gastroentestinal Clin North Am 2002; 31:307-27.

33. Weiss JS, Gupta AK, Regezi J, Rasmussen JE. Oral ulcers and cobblestone plaques. Oral Crohn.s disease. Arch Dermatol 1991; 127:889-8.

34. Gregory B, Ho VC. Cutaneous manifestations of gastrointestinal disorders: Part I, J Am Acad Dermatol 1992; 26:153-166.

35. Sweet Rd. An acute febrile neutrophilic dermatosis. Br J Dermatol 1964; 76:349.

36. Lam A, Borda IT, Inwood MJ, Thomson S. Coagulation studies in ulcerative colitis and Crohn's disease. Gastroenterology 1975; 68:245-51.

37. Ilan Y, Hillman M, Oran $\mathrm{R}$ et al. Vasculitis and cryoglobulinemia associated with persisting cholestatic hepatitis A virus infection. Am J Gastroenterol 1990;85:586-587.

38. Minkowitz G, Smoller BR, Mc Nutt NS. Benign cutaneous polyarteritis nodosa: Relationship to systemic polyarteritis nodosa and to hepatitis B infection. Arch Dermatol 1991; I 7:1520-1523.

39. Gocke DJ. Morgan C, Lockshin M et al. Association between polyarteritis and Australian antigen Lancet1970; 2:1149-1153.

40. Brouet JC, Clauvel JP, Danon F, Klein M, Seligmann M. Biologic and clinical significance of cryoglobulins. A report of 86 cases. Am J Med. 1974 Nov; 57(5):775-88.

41. Caputo R, Gelmetti C, Ermacora E et al. Gianotti-Crosti syndrome: a retrospective analysis of 308 cases. J Am Acad Dermatol. 1992; 26:207-210.

42. Angelo V, Chung RT, Kaplan LM. A role for hepatitis C virus in type II cryoglobinemia. N Engl J Med 1992; 327:1490-1495.

43. Bloch KJ. Cryoglobulinemia and hepatitis C virus. N Engl J Med1992; 327:1521-1522.

44. Barrace T, Corsini G, Granceschini R et al. Lichen planus induced by interferom-alpha-2a therapy for chronic active hepatitis C. Eur J Gastroenterol Hepatol 1995; 4:367-368.

45. Takikawa H, Yamazaki R, Shoji $\mathrm{S}$ et al. Normalization of urinary porphyrin level and disappearance of skin lesions after successful interferon therapy in a case of chronic hepatitis $\mathrm{C}$ complicated with porphyria cutanea tarda. J Hepatol 1995; 22:249-250.

46. Khanna VJ, Shieh S, Benjamin J et al. Necrolytic acral erythema associated with hepatitis C: effective treatment with interferon alfa and zinc. Arch Dermatol. Jun 2000;136(6):755-7.

47. Bonkovsky HL, Mehta S. Hepatitis C. A review and update. J Am Acad Dermatol. Feb 2001;44(2):159-82.

48. Reed JR, De Luca N, McIntyre AS et al. Localized morphea, xanthomatosis and primary biliary cirrhosis. Br J Dermatol 143:652

49. Chu CY, Yang CY, Huang SF et al. Lichen planus with xanthomatous change in a patient with primary biliary cirrhosis. Br J Dermatol 2000;142: 377-378.

50. Evstaf'ev VV, Levin MM. Dermatologic pathology in chronic alcoholic. Vestn Dermatol Venerol 1989; 8:72-74.

51. Satoh T, Xu R, Nishioka K. Vascular spiders and paper money skin improved by hemodialysis. Dermatology 2002; 205:76-74. 
52. Afford SC, Fisher NC, Neil D A et al. Distinct patterns of chemokine expression are associated wiyh leukocyte recruitment in alcoholic hepatitis and alcoholic cirrhosis. J Pathol 1998; 186:8289.

53. Schmucker DL, Ohta M, Kania S. Hepatic injury induced by bile salts: Correlation between biochemical and morphological events. Hepatology 1990; 12:1216-1221.

54. Ghent CN, Bloomer JR, Klatskin G. Elevations in skin tissue levels of bile acids in human cholestasis: Relation to serum levels and to pruritus. Gastroenterology 1977; 73:1125-1129.

55. Waalen J, Felitti V, Gelbart T, Ho Nj, Beutler E. Prevalence of hemochromatosis - related symptoms among individuals with mutations in the HFE gene. May Clin Proc 2002; 77:522-530

56. Seyhan M, Erdem T, Selimoğlu MA, Ertekin V. Dermatological signs in Wilson's disease. Pediatr Int. 2009 Jun;51(3):395-8.

57. Sibrack LA, Gouterman IH. Cutaneous manifestations of pancreatic diseases. Cutis. 1978 Jun;21(6):763-8.

58. Ozakyol AH, Sariçam T, Pasaoglu O. A rare entity: Cutaneous metastasis from gastric adenocarcinoma. Am J Gastroenterol.1999; 94:1118-9.

59. Andreev VC. Acanthosis nigricans. Stomatologia. 1963; 11:5 (in Bulgarian).

60. Brown J, Winkelmann RK. Review Acanthosis nigricans: a study of 90 cases. Medicine (Baltimore). 1968 Jan; 47(1):33-51.

61. Bell HK, Poston GJ, Vora J, Wilson NJ. Cutaneous manifestations of the malignant carcinoid syndrome. Br J Dermatol. 2005 Jan;152(1):71-5.

62. Van Beek AP, de Haas ER, van Vloten WA, Lips CJ, Roijers JF, Canninga-van Dijk MR . The glucagonoma syndrome and necrolytic migratory erythema: a clinical review". Eur. J. Endocrinol.Nov 2004; 151 (5): 531-7.

63. Gardner EJ. Follow-up study of a family group exhibiting dominant inheritance for a syndrome including intestinal polyps, osteomas, fibromas and epidermal cysts. Am J Hum Genet 1962; $14: 376$.

64. Gardner EJ, Richards RC. Multiple cutaneous and subcutaneous lesions occurring simultaneously with hereditary polyposis and osteomatosis. Am J Hum Genet 1953; 5:139.

65. Jeghers H, McCusick VA, Katz KH. Generalized intestinal polyposis and melanin spots of the oralmucosa, lips and digits. A syndrome of diagnostic significance. N Engl J Med 1949; 241: 993 1005.

66. Tomlinson IP, Houlston RS. Peutz-Jeghers syndrome. J Med Genet 1997; 34 (12): 1007-11.

67. Cronkhite LW Jr, Canada WJ. Generalized gastrointestinal polyposis; an unusual syndrome of polyposis, pigmentation, alopecia and onychotrophia. N Engl J Med 1955, 252:1011-1015.

68. Ward EM, Wolfsen HC. Review article: the non-inherited gastrointestinal polyposis syndromes. Aliment Pharmacol Ther 2002, 16:333-342.

69. Albrecht S, Haber RM, Goodman JC and Duvic M. Cowden Syndrome and L'hermitte- Duclos Disease. Cancer 1992; 70:869-76.

70. Marra G, Armelao F, Vechio FM, Percepe A, Anti M. Cowden disease with extensive gastrointestinal polyposis. J Clin Gastroenterol 1994; 18:42-7.

71. Akhtar S, Oza K, Khan S, et al. Muir-Torre syndrome: case report of a patient with concurrent jejunal and ureteral cancer and a review of literature. J Am Acad Dermatol. 1999;41:681-686. 
72. Schwartz RA, Torre DP. The Muir-Torre syndrome: a 25-year retrospect. J Am Acad Dermatol. 1995; 33:90-104.

73. Brandt T. Dermatitis in children with disturbances ofgeneral condition and absorption of food. Acta derm venereol (Stockh) 1936; 17:513-46.

74. Weismann K, Hoe S, Kauden L et al. Zinc absorption in patients suffering from acrodermatitis enteropathica and in normal adults assessed by whole - body c.

\section{AUTHORS:}

1. Manisha Nijhawan

2. Puneet Agarwal

3. Sandeep Nijhawan

4. Prashant

5. Abhishek Saini

\section{PARTICULARS OF CONTRIBUTORS:}

1. Associate Professor, Department of Skin and VD, Mahatma Gandhi Medical College \& Hospital, Jaipur, Rajasthan.

2. Resident Doctor, Department of Skin and VD, Mahatma Gandhi Medical College \& Hospital, Jaipur, Rajasthan.

3. Professor and Head, Department of Gastroenterology, SMS Medical College \& Hospital, Jaipur, Rajasthan.

4. Resident Doctor, Department of Gastroenterology, SMS Medical College \& Hospital, Jaipur, Rajasthan.
5. Resident Doctor, Department of Pediatrics, Mahatma Gandhi Medical College \& Hospital, Jaipur, Rajasthan.

\section{NAME ADDRESS EMAIL ID OF THE CORRESPONDING AUTHOR:}

Dr. Manisha Nijhawan, 112, Panscheel Enclave, JLN Road, Jaipur, Rajasthan.

E-mail:m.nijhawan12@gmail.com

Date of Submission: 27/01/2014. Date of Peer Review: 28/01/2014. Date of Acceptance: 14/02/2014. Date of Publishing: 01/03/2014. 